Somnologie $2016 \cdot 20: 159$

DOI 10.1007/s11818-016-0081-5

Online publiziert: 30. August 2016

๑) Springer-Verlag Berlin Heidelberg 2016

CrossMark

\author{
Angelika A. Schlarb ${ }^{1}$ Barbara Schneider ${ }^{2} \cdot$ Michael Arzt $^{3}$ \\ 'Fakultät für Psychologie und Sportwissenschaft, Universität Bielefeld, Bielefeld, Deutschland \\ ${ }^{2}$ Zentrum für Neuropädiatrie und Schlafmedizin, Kinderkrankenhaus St. Marien, Landshut, Deutschland \\ ${ }^{3}$ Schlafmedizinisches Zentrum der Klinik und Poliklinik für Innere Medizin II, Pneumologie, \\ Universitätsklinikum Regensburg, Regensburg, Deutschland
}

\title{
Pädiatrische Schlafmedizin und -forschung
}

graphischen Daten im Kinderschlaflabor. Wiederum andere Beiträge berücksichtigen und untersuchen vor allem klinische Themen und Störungen, und schließlich konzentrieren sich weitere Arbeiten auf die Unterschiede in den verschiedenen Altersgruppen. Auf diese Weise ist ein Heft entstanden, in dem sich das breite Spektrum der pädiatrischen Schlafmedizin und Schlafforschung widerspiegelt und das dazu einladen soll, das herausfordernde und spannende Forschungsund Betätigungsfeld rund um den Kinderschlaf besser kennenzulernen.

Wir als Herausgeber hoffen, dass dieses Schwerpunktheft Pädiatrie für die Leserschaft bereichernd ist und zu weiterer Forschung anregt. Allen Leserinnen und Lesern wünschen wir eine unterhaltsame, lehrreiche und spannende Lektüre.

Wir möchten allen Autoren und Reviewern, die zum Gelingen dieses Schwerpunkthefts beigetragen haben, für ihr großes Engagement und ihren Enthusiasmus außerordentlich danken.

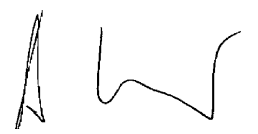

Angelika A. Schlarb

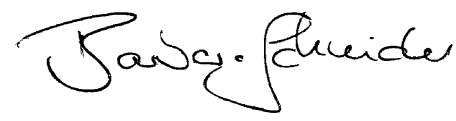

Barbara Schneider

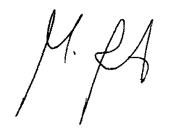

Michael Arzt
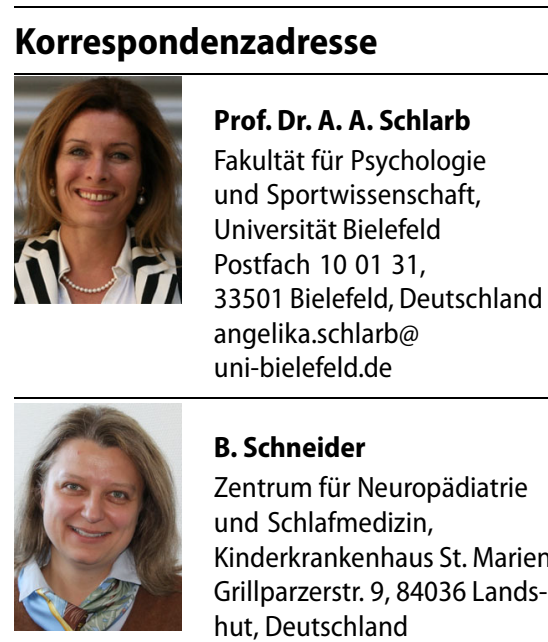

\section{B. Schneider}

Zentrum für Neuropädiatrie und Schlafmedizin,

Kinderkrankenhaus St. Marien Grillparzerstr. 9, 84036 Lands-

hut, Deutschland Barbara.Schneider@ st-marien-la.de

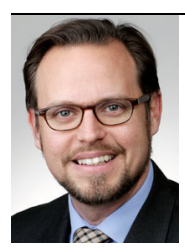

Prof. Dr. M. Arzt

Schlafmedizinisches Zentrum der Klinik und Poliklinik für Innere Medizin II, Pneumologie, Universitätsklinikum Regensburg Franz-Josef-Strauß-Allee 11, 93053 Regensburg, Deutschland Michael.Arzt@klinik. uniregensburg.de

Interessenkonflikt. A.A. Schlarb, B. Schneider und M. Arzt geben an, dass kein Interessenkonflikt besteht. fokussieren systemische Aspekte, die die familiäre Situation berücksichtigen, denn der Schlaf bei Säuglingen oder Kleinkindern kann nicht vollständig unabhängig vom familiären System gesehen werden. Andere wiederum fokussieren eher auf alters- und entwicklungsbezogene Aspekte. So stehen wir beispielsweise für alle Altersgruppen vor diagnostischen Herausforderungen wie der spezifischen Auswertung von polysomno- 